\title{
Decisão compartilhada na atenção primária e desfechos em saúde: uma revisão integrativa
}

\author{
Shared decision making in primary care and health outcomes: an integrative review \\ Decisión compartida en atención primaria y resultados en salud: una revisión integradora
}

\author{
Gabriel Glebocki $^{1 \oplus}$, Felipe G. Corneau' ${ }^{1 \oplus}$ \\ ${ }^{1}$ Secretaria de Saúde de São Bernardo do Campo
}

\section{Resumo}

O processo de decisão compartilhada pode ser definido a partir dos seguintes elementos: 1. há, no mínimo, duas pessoas envolvidas no processo de decisão, o médico e o paciente; 2 . médico e paciente compartilham informações; 3 . ambos contribuem para o processo decisório expondo suas preferências; 4 . chega-se a uma decisão sobre a qual todos os envolvidos concordam. Seu emprego se justifica principalmente pelo aspecto ético de incluir o paciente nas decisões cujas consequências ele sofrerá. Todavia, muito se questiona sobre a relação desta prática com os desfechos em saúde. Objetivos: Esse estudo tem por objetivo avaliar a relação entre a prática da decisão compartilhada e desfechos em saúde em cenários de atenção primária à saúde. Métodos: Realizou-se uma revisão integrativa da literatura e foram incluídos artigos que tivessem medidas empíricas de decisão compartilhada durante o encontro clínico, cujo cenário fosse a atenção primária à saúde e que apresentasse avaliação de, pelo menos, um desfecho em saúde. Resultados: Inclui-se dez artigos no estudo, e os temas abordados são depressão (4 artigos), hipertensão (2), diabetes (1), risco cardiovascular (1), rastreio de câncer colorretal (1) e infertilidade (1). Metade dos estudos sobre depressão encontraram associação positiva entre a decisão compartilhada e a melhora dos sintomas depressivos. Dos estudos sobre hipertensão, não se encontrou associações estatisticamente significativas. Do estudo sobre diabetes, não se constatou correlação positiva entre decisão compartilhada e redução da hemoglobina glicada e do LDL. Compartilhar a decisão ao discutir risco cardiovascular não piorou o escore deste indicador 6 meses após a consulta. Com relação ao rastreio de câncer colorretal, discutir riscos e benefícios e avaliar as preferências dos pacientes se associou negativamente à realização dos testes de rastreio. Por fim, decisão compartilhada se associou a melhor experiência de cuidado para pessoas em acompanhamento para infertilidade na atenção primária. Dois estudos cronometraram consultas e não se observou diferenças de tempo entre aqueles que usaram e os que não usaram a decisão compartilhada. Quatro estudos não definiram conceitualmente a decisão compartilhada e quatro estudos não utilizaram ferramentas validadas para medi-la. Conclusão: Com relação aos desfechos avaliados, os artigos incluídos nesta revisão apresentam resultados ambíguos, com aparente tendência de correlação positiva entre decisão compartilhada e desfechos. Todavia, a falta de uniformidade com relação à definição conceitual de decisão compartilhada parece ser potencial barreira para pesquisas de maior qualidade na área.

Palavras-chave: Tomada de Decisão Clínica, Atenção Primária à Saúde, Avaliação de Resultados da Assistência ao Paciente, Avaliação de Processos e Resultados em Cuidados de Saúde.

Como citar: Glebocki G, Corneau FG. Decisão compartihada na atenção primária e desfechos em saúde: uma revisão integrativa. Rev Bras Med Fam Comunidade. 2021;16(43):2388. https://doi.org/10.5712/rbmfc16(43)2388

\author{
Autor correspondente: \\ Gabriel Glebocki. \\ E-mail: gglebocki@gmail.com \\ Fonte de financiamento: \\ não se aplica. \\ Parecer CEP: \\ não se aplica. \\ Procedência: \\ não encomendado. \\ Avaliação por pares: \\ externa. \\ Recebido em: 11/02/2020. \\ Aprovado em: 10/06/2021.
}




\begin{abstract}
Introduction: The process of shared decision making can be defined through the following elements: 1. there are, at least, two persons involved in the decisional process, the doctor and the patient; 2 . doctor and patient share information; 3 . both contribute to the decisional processes exposing it's preferences; 4. a decision upon which all agree is achieved. Its use is justified mainly by the ethical aspect of including the patient in the decisions whose consequences he will suffer. However, much is questioned about the relation between this practice and health outcomes. Objectives: This study aims to evaluate the relation between shared decision making and health outcomes in primary care settings. Methods: An integrative review of the literature was carried out. Articles that contained an empirical measure of shared decision during the clinical encounter, whose scenario was primary health care and that presented evaluation of at least one health outcome were included. Results: Ten articles were included in the study, and the topics covered are depression (4 articles), hypertension (2), diabetes (1), cardiovascular risk (1), colorectal cancer screening (1), and infertility (1). Half of the studies on depression found a positive association between shared decision making and improvement of depressive symptoms. None of the studies on hypertension detected statistically significant associations. The diabetes study found a positive correlation between shared decision making and reduced glycated hemoglobin and LDL. Sharing the decision when discussing cardiovascular risk did not worsen the score of this indicator after 6 months. Regarding colorectal cancer screening, discussing risks and benefits and assessing patient preferences was negatively associated with the performance of screening tests. Finally, shared decision making was associated with better care experience for people being monitored for infertility in primary care. Two studies timed consultations and found no time differences between those who used and those who did not use shared decision making. Four studies did not conceptually define shared decision making and four studies did not use validated tools to measure it. Conclusion: Regarding the specified outcomes, the articles included in this review show ambiguous results, with an apparent positive correlation trend between shared decisions and outcomes. However, the lack of uniformity regarding the conceptual definition of shared decision making seems to be a potential barrier for higher quality research in the area.
\end{abstract}

Keywords: Clinical Decision-Making, Primary Health Care, Patient Outcome Assessment, Outcome and Process Assessment, Health Care

\title{
Resumen
}

Introducción: Se puede definir el proceso de decisión compartida a partir de los siguientes elementos: 1. hay al menos dos personas involucradas en el proceso de decisión, el médico y el paciente; 2. médico y paciente comparten información; 3. ambos contribuyen al proceso de toma de decisiones al exponer sus preferencias; 4 . se llega a una decisión sobre la cual todos los involucrados están de acuerdo. Su uso se justifica principalmente por el aspecto ético de incluir al paciente en las decisiones cuyas consecuencias sufrirá. Sin embargo, se cuestiona mucho la relación de esta práctica con los resultados de salud. Objetivo: Este estudio tiene como objetivo evaluar la relación entre la práctica de la decisión compartida y los resultados de salud en entornos de atención primaria de salud. Métodos: Se realizó una revisión integradora de la literatura. Se incluyeron artículos que contenían una medida empírica de decisión compartida durante el encuentro clínico, cuyo escenario era la atención primaria de salud y que presentaban una evaluación de al menos un resultado de salud. Resultados: Se incluyeron diez artículos en el estudio, y los temas cubiertos son depresión (4 artículos), hipertensión (2), diabetes (1), riesgo cardiovascular (1), detección del cáncer colorrectal (1) e infertilidad (1). La mitad de los estudios sobre depresión encontraron una asociación positiva entre la decisión compartida y la mejora de los síntomas depresivos. Ninguno de los estudios sobre hipertensión detectó asociaciones estadísticamente significativas. El estudio de diabetes encontró una correlación positiva entre la decisión compartida y la reducción de la hemoglobina glucosilada y el LDL. Compartir la decisión al discutir el riesgo cardiovascular no empeoró la puntuación de este indicador después de 6 meses. Con respecto a la detección del cáncer colorrectal, discutir los riesgos y beneficios y evaluar las preferencias del paciente se asoció negativamente con la realización de las pruebas de detección. Finalmente, decisión compartida se asoció con la mejor experiencia de atención para las personas que están siendo monitoreadas por infertilidad en atención primaria. Dos estudios cronometraron las citas y no encontraron diferencias de tiempo entre los que usaron y los que no usaron la decisión compartida. Cuatro estudios no definieron conceptualmente la decisión compartida y cuatro estudios no utilizaron herramientas validadas para medirla. Conclusión: Con respecto a los resultados evaluados, los artículos incluidos en esta revisión presentan resultados ambiguos, con una tendencia aparente de correlación positiva entre la decisión compartida y los resultados de salud. Sin embargo, la falta de uniformidad con respecto a la definición conceptual de la decisión compartida parece ser una barrera potencial para investigación de mayor calidad en el área.

Palabras clave: Toma de Decisiones Clínicas, Atención Primaria de Salud, Evaluación del Resultado de la Atención al Paciente, Evaluación de Procesos y Resultados en Atención de Salud

\section{INTRODUÇÃO}

\section{A definição de decisão compartilhada}

Há diversos modelos teóricos sobre como se dá a relação entre médico e paciente no encontro clínico. Charles et al. (1997), ${ }^{1}$ em artigo clássico sobre decisão compartilhada (DC), dividem essa relação em quatro principais tipos.

No modelo paternalista, todo o poder decisório reside no médico, que toma as decisões de maneira unilateral, sem considerar as preferências e valores do paciente. No modelo denominado decisão informada, o médico age como um consultor, transmitindo informações ao paciente que, por sua vez, é o único responsável pelas decisões. O modelo do médico como agente é aquele no qual o médico procura entender os valores e preferências do paciente para decidir o que ele, médico, julga ser o melhor caminho a seguir. 
Por fim, os autores definem o modelo de DC a partir de 4 componentes: 1. há, no mínimo, duas pessoas envolvidas no processo de decisão sobre o tratamento, o médico e o paciente; 2. médico e paciente compartilham informações entre si; 3. ambos contribuem para o processo decisório expondo suas preferências; 4 . chega-se a uma decisão sobre o tratamento que todos os envolvidos concordam. ${ }^{1}$

Ainda que não exista consenso acerca da definição conceitual sobre $\mathrm{DC},{ }^{2,3}$ e que alguns autores defendam conceitualizações mais abrangentes, ${ }^{4}$ a definição acima é amplamente aceita e já foi operacionalizada em instrumentos de medida.

\section{Justificativas para o emprego da DC}

Há três principais justificativas para a prática da DC nos encontros clínicos. Primeiro, considera-se eticamente desejável que o paciente possa decidir a respeito de intervenções sobre seu próprio corpo. A autonomia do paciente, que pode ser entendida como a obrigação por respeitar a capacidade de tomada de decisão de pessoas autônomas, ${ }^{5}$ é princípio bioético amplamente aceito e presente no código de ética médica brasileiro. ${ }^{6}$

Em segundo lugar, argumenta-se que a DC seria uma possível maneira de reduzir as demandas induzidas pelos profissionais de saúde. Wennberg et al. (1982) ${ }^{7}$ publicaram um estudo que demonstrava que parte significativa da variabilidade da prática médica (procedimentos cirúrgicos, no caso) entre localidades com características similares era explicada pela diferença na quantidade de especialistas presentes em cada área, pela avaliação que faziam dos pacientes (diagnósticos) e pelo valor que atribuíam às terapêuticas. Demonstraram que não era a prevalência de doenças, nem os valores e preferências dos pacientes que explicavam a variabilidade da prática médica. ${ }^{7} \mathrm{~A}$ partir desse diagnóstico, os autores ressaltaram a importância de incluir os pacientes nas decisões que, até então, variavam a despeito de suas preferências. ${ }^{8}$

Em terceiro lugar, pode-se considerar a prática da DC como elemento essencial na prática da medicina baseada em evidências. Elwyn e McCormack (2018), ${ }^{9}$ defendem que DC é um importante desfecho ao se avaliar prática médica baseada em evidências. Segundo os autores, é um erro avaliar a prática medicina baseada em evidências a partir de desfechos de saúde (valor da pressão, medida da glicemia, etc.) ou de desfechos populacionais (mortalidade, morbidade, etc.). Dado que compartilhar a decisão é fundamental, pode-se assumir que pessoas diferentes farão escolhas diferentes, o que resultarão em desfechos diferentes. Desse modo, o desfecho comum a ser avaliado é o grau de participação do paciente na decisão feita. ${ }^{9}$

Por fim, sob a perspectiva da medicina de família e comunidade (MFC), a decisão compartilhada é parte essencial do método clínico que serve de base para a especialidade. No livro "Medicina centrada na pessoa, transformando o método clínico" - amplamente utilizado na formação em MFC, Stewart afirma que "o modelo de tomada de decisão compartilhada e o método clínico centrado na pessoa [MCCP] se alinham de forma mais clara no terceiro componente", ainda que ressalte que não são sinônimos. ${ }^{10}$ Outra referência teórica importante para a MFC, que é o guia Calgary-Cambridge para entrevista médica, também destaca a necessidade da decisão compartilhada. ${ }^{11}$

\section{DC no Brasil e no mundo}

As pesquisas sobre DC têm crescido nos últimos anos e diversos países tem feito esforços para incentivar seu uso na prática clínica. ${ }^{12}$ Reino Unido e Austrália são exemplos de países que publicaram documentos oficiais que estimulam a prática da DC em seus respectivos sistemas de saúde. ${ }^{13,14}$ 
No Brasil, a Política Nacional de Humanização e a Política Nacional de Atenção Básica de 2017 tocam indiretamente no tema, sem, contudo, nomear DC como um objetivo concreto a ser atingido. ${ }^{15,16} \mathrm{~A}$ produção científica nacional acerca do tema ainda é incipiente. Uma busca no PubMed, em novembro de 2019, associando os termos "shared decision making" e "Brazil" no título ou resumo retornou apenas 9 resultados. A mesma busca, trocando o termo "Brazil" por "United States", retornou 302 artigos.

\section{Como medir DC}

O processo da DC pode ser dividido em três etapas: a primeira se refere ao desejo do paciente de participar ativamente das decisões; a segunda etapa se relaciona com o processo decisório no momento do encontro clínico; a terceira etapa corresponde a satisfação acerca do processo decisório. ${ }^{17}$

O processo decisório (segunda etapa), foco desta revisão, pode ser avaliado de três maneiras distintas: a primeira consiste na gravação da consulta e sua posterior avaliação a partir de instrumento desenhado para esta finalidade; a segunda maneira consiste na avaliação a partir da percepção do paciente, com o uso de questionários ou entrevistas; o terceiro modo de avaliação, à semelhança do anterior, se baseia em questionário ou entrevista, mas aplicados ao profissional de saúde. ${ }^{17}$

Em 2011, uma revisão sobre os instrumentos disponíveis para medir DC encontrou 8 instrumentos para avaliar antecedentes da decisão, 12 para o processo de decisão na consulta e 9 instrumentos cujo foco eram os desfechos relacionados à decisão. ${ }^{17}$

\section{OBJETIVOS}

Ainda que a melhora de desfechos clínicos não seja a motivação principal para adoção da DC, é relevante avaliar qual o seu efeito nesse campo, particularmente por ser prática frequentemente incentivada sob este pretexto. ${ }^{18}$ Esta revisão tem como objetivo avaliar a relação entre o processo de DC - em cenários de atenção primária à saúde (APS) - e desfechos em saúde.

\section{MÉTODOS}

Este estudo é uma revisão integrativa. ${ }^{19}$ As buscas foram realizadas no dia 28 de setembro de 2019, sem limite de data de publicação e foram pesquisadas as bases disponíveis via PubMed e BVS. Em ambas, o objetivo era a intersecção de três campos: DC durante o encontro clínico, APS e desfechos em saúde. Devido à restrição de tempo, foram utilizadas buscas mais restritivas. As frases de busca estão apresentadas no Quadro 1.

Quadro 1. Frases de busca utilizadas.

\begin{tabular}{|c|c|}
\hline PubMed & VVS \\
\hline $\begin{array}{l}\text { ("Primary Health Care" [Mesh:noexp]) } \\
\text { AND ("Shared Decision Making" OR "Shared } \\
\text { Decision-Making" OR "Patient Participation" [Mesh]) } \\
\text { AND ("Outcome Assessment (Health Care)" [Mesh] } \\
\text { OR "Pregnancy Outcome" [Mesh] OR "Fatal Outcome" } \\
\text { [MESH] OR "Numbers Needed To Treat" [Mesh] OR } \\
\text { "Health Outcome" OR "Healthcare outcome") }\end{array}$ & $\begin{array}{l}\text { (mh:(“Tomada de Decisões”) OR tw:(“decisão compartilhada”) OR } \\
\text { mh:(“participação do paciente”)) AND (mh:(“Atenção Primária à Saúde”) OR } \\
\text { tw:(“Atenção Básica”) OR tw:(“Atenção Básica à Saúde”)) } \\
\text { AND (mh:(“Medidas de Associação, Exposição, Risco ou Desfecho”) OR } \\
\text { mh:(“Avaliação de Processos e Resultados (Cuidados de Saúde)”) OR } \\
\text { mh:(“Resultado do Tratamento”) OR mh:(“Avaliação de Resultados (Cuidados } \\
\text { de Saúde)") OR mh:(“Avaliação de Resultados da Assistência ao Paciente”)) }\end{array}$ \\
\hline
\end{tabular}


Foram incluídos artigos com presença de medida empírica de DC durante o encontro clínico - em oposição a medidas de antecedentes da decisão ou satisfação com a decisão, como definidos no artigo de Scholl et al. (2011) $)^{17}$-; cujo cenário fosse a APS; que tivessem medidas de pelo menos um desfecho de saúde; e cujo idioma fosse inglês ou português. Foram excluídos estudos que avaliassem exclusivamente antecedentes ou satisfação com as decisões ou que não apresentassem medidas empíricas de DC.

Os dados foram extraídos em planilha construída especificamente para este fim pelo autor do artigo.

\section{RESULTADOS}

\section{Características gerais dos artigos selecionados}

Foram identificados 110 artigos via PubMed e 129 via BVS, dos quais 76 apareciam em ambas as bases, resultando em 155 artigos. Foram excluídos 64 artigos a partir da leitura dos títulos e 66 artigos a partir da leitura dos resumos. Vinte e cinco artigos foram selecionados para leitura completa. Destes, 11 foram excluídos por não apresentarem medidas empíricas de DC, 5 foram excluídos por medirem antecedentes da decisão ou satisfação com a decisão, mas não o processo em si. Nove artigos foram incluídos para esta revisão.

Dentre os nove artigos selecionados, havia duas revisões sistemáticas, ${ }^{20,21}$ e ambas incluíam artigos que não avaliavam DC de maneira empírica, considerado critério de inclusão neste estudo. Optou-se por identificar, dentre os artigos que compunham estas revisões ( $7 \operatorname{artigos}^{20}$ e $\left.6 \operatorname{artigos}^{21}\right)$, aqueles cujo escopo estivesse adequado a esta revisão. Foram identificados três artigos que ainda não estavam presentes na seleção realizada. ${ }^{22-24}$ Estes foram incluídos na análise e as revisões sistemáticas excluídas. Ao final, dez artigos constituíram a base para este estudo.

Os artigos selecionados são provenientes da Alemanha, ${ }^{24-26}$ Estados Unidos ${ }^{22,27-30}$ e Inglaterra. ${ }^{31}$ As condições de saúde avaliadas são depressão, ${ }^{25-27,30}$ hipertensão, ${ }^{22,23}$ diabetes, ${ }^{29}$ risco cardiovascular, ${ }^{24}$ rastreio de câncer colorretal ${ }^{28}$ e infertilidade. ${ }^{31}$ Há apenas um estudo qualitativo. ${ }^{31} \mathrm{O}$ tamanho da amostra variou de 22 a 1.706 pessoas. ${ }^{27,31}$

A Tabela 1 apresenta um resumo sobre os artigos incluídos nesta revisão.

\section{Avaliação dos desfechos}

Dentre os estudos que avaliaram depressão, dois apontaram melhoras nos sintomas depressivos ${ }^{25,27}$ e dois não apontaram associação entre DC e desfechos clínicos. ${ }^{26,30}$ Nenhum dos estudos que avaliou hipertensão encontrou correlação entre DC e redução da pressão arterial. Um deles destacou que há indícios de redução da pressão arterial em pacientes hipertensos não controlados, porém, não encontrou significância estatística. ${ }^{22} \mathrm{O}$ outro estudo sobre hipertensão, embora não tenha encontrado redução da pressão arterial, também não apresentou diferença no nível de DC entre os grupos intervenção e controle. ${ }^{23}$ O estudo sobre diabetes encontrou correlação positiva entre DC e redução da hemoglobina glicada, bem como redução do LDL. Este estudo avaliou, também, pressão arterial, porém, não encontrou correlação entre pressão arterial e DC. ${ }^{29} \mathrm{O}$ risco cardiovascular não apresentou variação significativa entre o grupo que teve maior participação dos pacientes e mais DC se comparado ao grupo controle. ${ }^{24}$ 
Tabela 1. Descrição dos artigos incluídos nesta revisão.

\begin{tabular}{lcc}
\hline Autor / ano / país & Clever [27] / 2006 / Estados Unidos & Loh [25] / 2007 / Alemanha \\
\hline Tipo de estudo & Coorte prospectiva & $\begin{array}{c}\text { Observacional - survey na consulta inicial e } \\
6 \text { a 8 semanas após }\end{array}$ \\
\hline Condições avaliadas & Depressão & Depressão \\
\hline Objetivo & $\begin{array}{c}\text { Avaliar se DC }{ }^{1} \text { está associada a tratamento } \\
\text { concordante com guideline e com melhora } \\
\text { de sintomas depressivos. }\end{array}$ & $\begin{array}{c}\text { Avaliar o impacto da participação do } \\
\text { paciente na aderência ao tratamento e a } \\
\text { desfechos clínicos. }\end{array}$ \\
\hline
\end{tabular}

Dados foram extraídos de 4 ensaios clínicos randomizados independentes que tinham como objetivo aumentar o percentual de pacientes em tratamento adequado (concordante com guideline) para depressão. Nenhum dos estudos base tinha $D^{1}$ como intervenção. Seis meses após a $1^{\underline{a}}$ consulta, mediram DC ${ }^{1}$ a partir da seguinte pergunta: "(...) como você avaliaria seu envolvimento nas decisões sobre seu cuidado." Nessa ocasião, e a cada 6 meses (até completar 2 anos) acessaram também se tratamento estava de acordo com guidelines e sintomas depressivos.

- Envolvimento na tomada de decisão (reportado pelo paciente)

Variáveis analisadas (instrumento utilizado)
- Tratamento de acordo com guideline (reportado pelo paciente)

- Sintomas depressivos (PHQ-D8)
Trinta $\mathrm{mfc}^{3}$ selecionados por conveniência recrutaram pacientes com necessidade de tratamento para depressão. Foram aplicados questionários na primeira consulta e 6 a 8 semanas após. Foi medido participação do paciente, adesão ao tratamento e sintomas depressivos.
- Sintomas depressivos (CES-D2 modificada).

- Participação do paciente (reportado pelo paciente)

- Adesão ao tratamento (reportado pelo paciente e pelo médico)

\section{Conclusões principais}

- DC1 está associado a melhora de sintomas e a maior aderência ao guideline. Isso ocorreu tanto nos pacientes do grupo intervenção como no controle

- Aderência ao guideline não é um bom mediador para explicar a relação entre $D^{1}$ e melhora de sintomas. Sugere que DC ${ }^{1}$

atue na melhora dos sintomas por outra via

\begin{tabular}{|l}
\hline Define DC \\
\hline Questionário validado para DC \\
\hline Tamanho da amostra
\end{tabular}

Tamanho da amostra 


\begin{tabular}{|c|c|c|}
\hline Descrição breve & $\begin{array}{l}\text { Trinta mfc } \text { moram a unidade de } \text { for } \\
\text { randomização do estudo, divididos } \\
\text { em grupo controle e intervenção. Mfc } \\
\text { recrutaram pacientes com depressão } \\
\text { recém diagnosticada com necessidade de } \\
\text { tratamento. Foi realizado um questionário } \\
\text { após a primeira consulta e outro } 6 \text { a } 8 \\
\text { semanas após. Os médicos do grupo } \\
\text { intervenção receberam treinamento em } \\
\text { DC e o processo se repetiu com novos } \\
\text { pacientes. Os questionários avaliaram } \\
\text { DC }{ }^{1} \text {, adesão ao tratamento e sintomas } \\
\text { depressivos. O tempo de consulta também } \\
\text { foi medido. }\end{array}$ & $\begin{array}{l}\text { Gravaram consultas de pacientes que } \\
\text { tiveram testes de rastreio para câncer } \\
\text { colorretal solicitados. Avaliaram a } \\
\text { ocorrência ou não } \mathrm{DC}^{1} \text { nas gravações e } \\
\text { correlacionaram com a realização ou não } \\
\text { do teste pelos pacientes. }\end{array}$ \\
\hline $\begin{array}{l}\text { Variáveis analisadas (instrumento } \\
\text { utilizado) }\end{array}$ & $\begin{array}{c}\text { - Sintomas depressivos }\left(\mathrm{PHQ}-\mathrm{D}^{8}\right) \\
\text { - Participação do paciente }\left(\mathrm{MSH}^{9}\right) \\
\text { DC }^{1}\left(\mathrm{PICS}^{10}\right) \\
\left.\text { - Satisfação do paciente (CSQ-8 }{ }^{11}\right) \\
\text { - Adesão ao tratamento (reportado pelo } \\
\text { paciente e pelo médico) } \\
\text { - Tempo de consulta }\end{array}$ & $\begin{array}{l}\text { - Presença de elementos de DC1 } \\
\text { - Realização de teste de rastreio para } \\
\text { câncer de cólon }\end{array}$ \\
\hline Define DC & Sim & Sim \\
\hline Questionário validado para DC & $\begin{array}{l}\cdot \mathrm{PICS}^{10} \\
\cdot \mathrm{MSH}^{9}\end{array}$ & Não \\
\hline Tamanho da amostra & 405 & 91 \\
\hline Conclusões principais & $\begin{array}{l}\text { - Grupo intervenção apresentou aumento } \\
\text { da DC1 e da satisfação do paciente } \\
\text { - Não houve diferença entre os grupos com } \\
\text { relação a tempo de consulta, adesão ao } \\
\text { tratamento e melhora clínica }\end{array}$ & $\begin{array}{c}\text { • Faz-se pouca DC }{ }^{1} \text { no geral } \\
\text { - Associação positiva entre: checar } \\
\text { entendimento do paciente e realização do } \\
\text { rastreio } \\
\text { - Associação negativa entre: discutir prós } \\
\text { e contras e realização do rastreio; discutir } \\
\text { preferências do paciente e realização do } \\
\text { rastreio. } \\
\text { - Já ter realizado teste de rastreio em } \\
\text { outras ocasiões pode ter sido fator de } \\
\text { confusão relevante, dado que este era o } \\
\text { fator que mais explicava a realização de } \\
\text { novo teste }\end{array}$ \\
\hline
\end{tabular}

\section{Autor / ano / país

Tipo de estudo

\section{Objetivo}

\section{Descrição breve}

Trinta $\mathrm{mfc}^{3}$ foram a unidade de randomização do estudo, divididos em grupo controle e intervenção. Mfc recrutaram pacientes com depressão recém diagnosticada com necessidade de tratamento. Foi realizado um questionário após a primeira consulta e outro 6 a 8 semanas após. Os médicos do grupo DC1 1 adesão ao tratamento e sintomas foi medido.

- Sintomas depressivos (PHQ-D $\left.{ }^{8}\right)$ - DC ${ }^{1}\left(\right.$ PICS $\left.^{10}\right)$

- Satisfação do paciente (CSQ-8 ${ }^{11}$ ) paciente e pelo médico)

po de consulta
- PICS

MSH

\section{Krones [24] / 2008 / Alemanha}

Ensaio clínico randomizado em cluster

Determinar o efeito da comunicação efetiva do risco cardiovascular e da $\mathrm{DC}^{1}$ a partir de ferramenta simples de auxílio a decisão (ARRIBA-Herz) para uso por $\mathrm{mfc}^{3}$ na APS ${ }^{4}$.

Médicos foram randomizados para grupo intervenção e controle. A intervenção consistia em treinamento para uso de ferramenta de auxílio à decisão clínica sobre $\mathrm{RCV}^{7}$ e possíveis intervenções. $O$ grupo controle recebeu seminários sobre assuntos não relacionados a $\mathrm{RCV}^{7}$. Após as consultas, foi avaliada participação e satisfação do paciente, $\mathrm{DC}^{1}, \mathrm{RCV}^{7} \mathrm{e}$ conhecimento sobre RCV ${ }^{7}$. Após seis meses, foi medido arrependimento sobre a

\section{Wilkes [31] / 2009 / Inglaterra}

Qualitativo - entrevistas em profundidade

Explorar as razões subjacentes às atitudes e percepções relativas à experiência dos pacientes da APS 4 durante a abordagem da infertilidade. decisão e RCV? . 
.... Continuação

Variáveis analisadas (instrumento utilizado)

- Participação do paciente (Patient Participation Scale)

- Satisfação com a decisão - DC ${ }^{1}$ (SDM-Q-9)

- Conhecimento sobre prevenção CV

- Arrependimento com a decisão (decision regret scale)

- Risco CV (Score de Framingham)

\begin{tabular}{lcc}
\hline Define DC & Sim & Não \\
\hline Questionário validado para DC & SDM-Q-9 & Não \\
\hline Tamanho da amostra & 1132 & 22
\end{tabular}

- Intervenção aumentou participação e

satisfação do paciente, bem como DC ${ }^{1}$

- Intervenção reduziu arrependimento sobre a decisão

Conclusões principais

- DC ${ }^{1}$ não piora RCV ${ }^{7}$

- Ferramenta de auxílio a decisão sobre

$\mathrm{RCV}^{7}$ pode ser disseminada para médicos de família via sessões de educação continuada

\begin{tabular}{lc}
\hline Autor / ano / país & Parchman [29] / 2010 / Estados Unidos \\
\hline Tipo de estudo & Observacional - questionário na consulta \\
inicial e 12 meses após
\end{tabular}

\section{Condições avaliadas}

Objetivo

\section{Descrição breve}

Ao final, agruparam os achados nas seguintes categorias:

- Relações pessoais

- Relações profissionais

- Autonomia na tomada de decisão - Acesso aos serviços
- Uma boa experiência no manejo da infertilidade, dentre outras coisas, depende de abordagem centrada no paciente com profissional de saúde que incentiva tomada de decisões informadas pelo casal

- O estudo aborda diversos outros temas que fogem ao escopo deste estudo
Foram selecionados médicos da APS4 por conveniência. Todos seus pacientes portadores de diabetes receberam cartas convite para participar do estudo. Os que aceitaram, responderam um questionário base e um questionário após 12 meses. Os questionários avaliaram DC1, ativação do paciente e adesão ao tratamento. Também foram checados os registros do prontuário para avaliar pressão arterial, $\mathrm{LDL}^{5} \mathrm{e}$ $\mathrm{HbA} 1 \mathrm{c}^{6}$.
Avaliar uma possível relação causal entre $D^{1}$ e melhores desfechos clínicos, a partir da hipótese de que esta relação é mediada por maior aderência ao tratamento.

\section{Cooper [22] / 2011 / Estados Unidos}

Ensaio clínico randomizado Hipertensão

Comparar a efetividade de intervenções em médicos e pacientes - com baixo status socioeconômico - com o objetivo de melhorar a comunicação durante a consulta.

Médicos e pacientes foram randomizados para intervenção intensiva ou mínima. A intervenção intensiva para médicos consistia em feedback após consulta simulada com o objetivo de melhorar habilidades de comunicação. O grupo sob intervenção mínima participou de consulta simulada, porém, sem feedback. Todos os pacientes receberam 1 sessão sobre habilidades de comunicação na consulta. Os pacientes sob intervenção intensiva receberam fotonovelas a reforçando as mensagens do treinamento. As consultas simuladas dos médicos foram gravadas em vídeo e as consultas reais em áudio.

Gravações serviram para avaliar o comportamento dos médicos. Pacientes avaliaram o estilo de decisão dos médicos e seu envolvimento no cuidado a partir de questionários. Foi avaliada adesão ao tratamento e pressão arterial.

- Comportamento de comunicação do

médico (RIAS)
- Estilo de tomada de decisão do médico

- DC' (reportado pelo paciente)

\begin{abstract}
Variáveis analisadas (instrumento utilizado)
\end{abstract}

- Ativação do paciente (escala de comunicação de Lorig)

- Adesão ao tratamento (Morisky)

- LDL ${ }^{5}, \mathrm{HbA}_{1} \mathrm{c}^{6}$ e pressão arterial (reportado pelo paciente) - $\mathrm{DC}^{1}\left(\mathrm{PICS}^{10}\right)$

- Adesão ao tratamento (Morisky) - Pressão arterial

\section{Define DC \\ Questionário validado para DC} Sim Não Não Tamanho da amostra

$141 \quad 279$


.... Continuação

\begin{tabular}{|c|c|c|}
\hline Conclusões principais & $\begin{array}{c}\text { - Maior uso de } \mathrm{DC}^{1} \text { se associou a maior } \\
\text { participação do paciente no encontro } \\
\text { clínico } \\
\text { - Participação ativa do paciente se } \\
\text { associou positivamente a adesão ao } \\
\text { tratamento } \\
\text { - Maior adesão ao tratamento se associou } \\
\text { positivamente com redução de } \mathrm{LDL}^{5} \mathrm{e} \\
\text { HbA1c } \\
\text { - Os resultados acima não se mostraram } \\
\text { válidos para hipertensão arterial }\end{array}$ & $\begin{array}{c}\text { - As intervenções intensivas apresentaram } \\
\text { melhores resultados } \\
\text { - Houve melhora na comunicação e na DC } \\
\text { • Há indícios de redução da pressão } \\
\text { arterial em paciente hipertensos não } \\
\text { controlados }\end{array}$ \\
\hline Autor / ano / país & Tinsel [23] / 2013 / Alemanha & LeBlanc [30] / 2015 / Estados Unidos \\
\hline Tipo de estudo & Ensaio clínico randomizado em cluster & Ensaio clínico randomizado em cluster \\
\hline Condições avaliadas & Hipertensão & Depressão \\
\hline
\end{tabular}

Tinta e seis clínicas de APS $^{4}$ foram randomizadas para intervenção ou controle. A intervenção consistiu no treinamento dos $\mathrm{mfc}^{3}$ nos seguintes elementos: hipertensão arterial; comunicação médico paciente e

\section{Descrição breve}

comunicação de risco; passos da $\mathrm{DC}^{1}$; entrevista motivacional; introdução a

uma tabela de decisão que lista opções para reduzir risco cardiovascular.

Foram utilizados casos simulados para dramatizações de consultas. Foram realizadas medidas no momento do recrutamento, 6, 12 e 18 meses após.

- Pressão arterial, colesterol, $\mathrm{HbA}_{1 c^{6}}$ - DC ${ }^{1}$ (SDM-Q-9)

Variáveis analisadas
(instrumento utilizado)

- Adesão ao tratamento (MARS-D)

- Conhecimento sobre hipertensão por parte do paciente

Dez clínicas de APS ${ }^{4}$ foram randomizadas para usar ou não um instrumento de auxílio a decisão clínica (DMC) no tratamento de pacientes com depressão grave a moderada. Medidas relativas a qualidade da decisão foram realizadas por questionários após as consultas. DC1 foi avaliado a partir da gravação de consultas. Desfechos clínicos (sintomas depressivos) foram avaliados no início do estudo, 3 e 6 meses após. Aderência à medicação foi avaliada a partir de registros de farmácia.

- Conforto com a decisão (DCS $\left.{ }^{12}\right)$

- Sintomas depressivos (PHQ-98)

- Adesão ao tratamento (registros da farmácia) - DC1 (OPTION ${ }^{13}$ ) - Tempo de consulta

\begin{tabular}{l}
\hline Define DC \\
\hline Questionário validado \\
\hline Tamanho da amostra \\
\hline \\
Conclusões principais
\end{tabular}

Não

SDM-Q-9

1120

Não

OPTION $^{13}$

- O treinamento não aumentou DC1 (sob o ponto de vista dos pacientes), não contribuiu para redução dos níveis de pressão arterial, não melhorou adesão ao tratamento e não aumentou o conhecimento dos pacientes sobre hipertensão arterial

297

- Pacientes e médicos que usaram o DMC sentiram-se mais confortáveis com as decisões

- Não houve diferença nos sintomas depressivos

- O grupo intervenção fez mais DC1

- Não houve diferença no tempo de consulta
1. DC: decisão compartilhada
2. CES-D: Center for Epidemiological
Studies Depression Scale
3. mfc: médico de família e comunidade
4. APS: atenção primária à saúde
5. LDL: low density lipoprotein
6. HbA1c: hemoglobina glicosilada
7. RCV: risco cardiovascular
8. PHQ-D: patient health questionaire
9. MSH: escala de Man-Son-Hing
10. PICS: Perceived Involvement in Care Scale
11. CSQ-8: Client Satisfaction Qustionnaire
12. DCS: Decisional Conflict Scale
13. OPTION: Observing Patient Involvement 
Para investigar a relação entre DC e rastreio de câncer colorretal, Ling et al. $(2008)^{28}$ usaram como base o modelo de decisão informada desenvolvido por Braddock. Este modelo avalia nove diferentes aspectos da comunicação entre médico e paciente. Discutir riscos e benefícios e avaliar as preferências dos pacientes se associou negativamente à realização dos testes de rastreio. O estudo que avaliou o manejo da infertilidade na APS, a partir de entrevistas, destacou o incentivo à DC como fator relevante para uma boa experiência por parte dos pacientes. ${ }^{31}$

Com relação aos desfechos relacionados a experiência do paciente, quatro estudos apontaram correlação positiva entre DC e conforto com a decisão, ${ }^{26}$ satisfação com a decisão ${ }^{24,30}$ e experiência de cuidado. ${ }^{31} \mathrm{Um}$ estudo associou mais DC a menor arrependimento seis meses após a tomada de decisão. ${ }^{24}$

\section{Definição de DC e instrumentos para medida de DC}

Quatro artigos não explicitaram qual definição conceitual utilizaram para $\mathrm{DC}^{25,28,29,31}$ e quatro artigos não usaram questionário validado para medir DC. ${ }^{22,23,30,31}$ Apenas um artigo não definiu o conceito nem utilizou questionário validado sobre DC. ${ }^{31}$ Há cinco artigos que citam uma definição conceitual para DC, todavia, apenas dois deles - elaborados pelo mesmo autor principal ${ }^{25,26}$ - citam a mesma referência, os demais citam definições de autores distintos.

Dentre os artigos que utilizaram instrumentos validados para medir DC, três usaram medidas a partir de questionários ${ }^{22-24,26}$ e um utilizou medida observacional a partir da gravação de consultas. ${ }^{30}$ Os instrumentos subjetivos (respondidos por pacientes ou médicos) utilizados foram perceived involvement in care scale (PICS) e 9-item shared decision making questionnaire (SDM-Q-9). A escala observing patient involvement (OPTION) foi o instrumento observacional utilizado e consiste na aplicação do instrumento de avaliação sobre consultas gravadas.

\section{Tempo de consulta}

Dois artigos mediram tempo de consulta e nenhum deles encontrou diferenças significativas entre os grupos controle e intervenção. ${ }^{26,30}$

\section{DISCUSSÃO}

\section{Resumo dos principais achados}

A falta de uniformidade com relação a definição conceitual de DC - e sua ausência em cerca de metade dos artigos - reforça achados de artigos anteriores, que já apontavam para este fato como potencial barreira para pesquisas na área. ${ }^{2,3,20}$ Clayman e Makoul (2006) $)^{2}$ elencaram os 3 principais problemas relativos à falta de clareza conceitual sobre DC. Primeiro, há o risco de medidas de DC inconsistentes. Medidas inconsistentes, por sua vez, dificultam avaliar as relações entre DC e desfechos. Por fim, falta de conceituação clara e consensual torna muito mais difícil a comparação entre estudos. ${ }^{2}$

Quanto aos desfechos avaliados, os artigos incluídos nesta revisão apresentam resultados ambíguos, com aparente tendência de correlação positiva entre DC e desfechos, sejam eles clínicos ou reportados pelos pacientes. Não há, todavia, consenso entre os estudos e quase todos indicam necessidade de mais 
pesquisas na área. Exceto pelo resultado de um estudo - relacionado ao rastreio de câncer colorretal ${ }^{28}$ não há indícios de correlação negativa entre DC e desfechos, ou seja, ainda que não se tenha evidências suficientes para afirmar que DC melhora desfechos, pode-se, com maior grau de certeza, afirmar que não contribui para sua piora. Dado que a principal motivação para adoção da DC é o aspecto ético, o fato de não contribuir para piora de desfechos pode ser interpretado como argumento a favor de sua adoção.

Com relação às doenças abordadas, nota-se que 4 dos 10 artigos tratam sobre depressão, ${ }^{25-27,30}$ o que condiz com a crescente importância atribuída à saúde mental como causa de morbidade ${ }^{32}$ fato relevante para os profissionais da APS que, cada vez mais, lidam com problemas de saúde mental.

\section{Comparação com a literatura}

Uma revisão sistemática de 2008 obteve resultados semelhantes aos deste estudo, encontrando correlação positiva entre DC e desfechos em 5 de 11 artigos selecionados. ${ }^{33}$ Os autores concluem que DC pode ser efetiva e útil principalmente para decisões de longo prazo. Todavia, não há menção sobre quais os instrumentos utilizados para medir DC, uma limitação importante, pois abre espaço para estudos que testam intervenções que supostamente melhoram o processo de DC sem de fato avaliar se isso ocorre. Este risco fica claro no artigo de Tinsel et al. (2013), ${ }^{23}$ no qual a intervenção proposta para aumentar DC não se comprovou efetiva quando medida com instrumento adequado.

Shay e Lafata (2015) ${ }^{34}$ publicaram uma revisão intitulada "Where is the evidence? A systematic review of shared decision making and patient outcomes" na qual acharam correlação positiva entre decisão compartilhada e desfechos em $43 \%$ dos desfechos avaliados. Em sua revisão, dividiram os desfechos em 3 categorias: afetivo-cognitivos (atitudes, emoções e conhecimento), comportamentais (aderência ao tratamento) e fisiológicos/de saúde (medidas de qualidade de vida, medidas fisiológicas, tais como pressão arterial, etc.). Concluíram que os desfechos afetivo-cognitivos são os mais influenciados pelo processo de DC, e destacaram que apenas as medidas subjetivas de DC pelos pacientes se associaram a melhores desfechos fisiológicos/de saúde. Destacam, ao final, que sua revisão não encontrou estudos que correlacionassem medida observacional de DC e desfechos de saúde. ${ }^{34}$ A presente revisão incluiu um estudo que investigou o impacto da DC - via OPTION, uma medida observacional de DC - e depressão. Este estudo encontrou relação entre DC e conforto com a decisão, porém, o mesmo não ocorreu com relação aos sintomas depressivos, ${ }^{30}$ corroborando os achados da revisão realizada por Shay e Lafata (2015). ${ }^{34}$

Quanto às condições de saúde, vale ressaltar a prevalência de estudos sobre saúde mental. Nas revisões de $2008^{33}$ e de $2015,{ }^{35}$ o tema representou $18 \%$ e $10 \%$ dos artigos incluídos, respectivamente. Esta revisão incluiu 4 estudos sobre depressão, o que representa $40 \%$ do total. Este fato talvez possa ser explicado pela crescente importância atribuída a saúde mental como causa de morbidade ${ }^{32}$ fato relevante para os profissionais da APS que, cada vez mais, lidam com problemas de saúde mental.

Há duas características que diferenciam este estudo das revisões citadas acima: a primeira é que nenhuma delas tem foco exclusivo na APS; a segunda é que esta revisão tem interesse específico no processo de decisão compartilhada, distinguindo-o dos antecedentes da decisão e considerando satisfação com a decisão apenas como desfecho do processo decisório. Todavia, os resultados encontrados foram similares. 
Buscando uma perspectiva populacional, Hughes et al. (2018) ${ }^{36}$ incluíram questões sobre decisão compartilhada no questionário "Medical expenditures panel survey". Este questionário consiste em uma família de pesquisas com representatividade nacional (estadunidense) que tem por objetivo estimar gastos em saúde, utilização dos serviços de saúde, fontes de pagamento, etc. A partir de uma amostra de mais de 60 mil pessoas, concluíram que menos DC está associada a piores desfechos relatados pelos pacientes, piores indicadores de qualidade do cuidado e maior utilização do sistema de saúde. Identificaram, também, correlação entre percepção de DC e raça - com hispânicos, negros e asiáticos atribuindo piores notas se comparados aos brancos. Reportam também que pessoas com maior grau de escolaridade e mais ricos reportam pior experiência com DC do que os mais pobres e com menos escolaridade. ${ }^{36}$

Uma revisão realizada por Sanders et al. (2013), ${ }^{20}$ com foco na APS, achou resultados semelhantes aos do estudo supracitado. Destacam a pequena quantidade de artigos selecionados, especialmente se levada em conta a relevância do assunto, e atribuem isso, em parte, a falta de conceituação clara sobre DC. Ao final, sugerem que pesquisas na APS tenham como objeto de estudo sintomas e não doenças, e que os desfechos avaliados sejam orientados aos pacientes e não às doenças, dado que grande parte das consultas nesse cenário tem como motivo de consulta sintomas e não doenças. ${ }^{20}$

\section{Fortalezas e limitações do estudo e implicações para a prática e pesquisa}

Essa revisão apresenta limitações importantes. São elas: frases de busca muito restritas podem ter deixado de incluir artigos relevantes, como ficou demonstrado pelos três artigos extraídos das revisões sistemáticas excluídas deste estudo; o fato de apenas uma pessoa ter realizado as buscas e a extração dos dados abre espaço para vieses pessoais e perda de informações e, por fim, a limitação decorrente da não abordagem sistemática da qualidade dos artigos selecionados.

Em contrapartida, a DC é tema relevante para médicos de família e comunidade e ainda pouco pesquisado no Brasil. ${ }^{37}$ Ademais, não é infrequente na literatura artigos que atribuem à DC mais atributos positivos do que ela parece possuir, tais como redução de custos, redução de medicalização excessiva ou melhora de desfechos. ${ }^{18}$ Sob este aspecto, esta revisão tem como fortaleza uma visão crítica sobre os possíveis benefícios e riscos da DC na prática clínica na APS.

\section{CONCLUSÃO}

Essa revisão se propôs a avaliar a relação entre a prática de DC na APS e desfechos em saúde. De acordo com as evidências coletadas, ainda que pareça haver correlação positiva entre DC e desfechos, as evidências ainda são frágeis e há, ainda, necessidades de estudos maiores e de melhor qualidade. A falta de consenso em torno do conceito de decisão compartilhada aparece repetidamente como barreira importante para a evolução desse campo de pesquisa.

Todavia, é essencial destacar os principais motivos pelos quais a prática da decisão compartilhada ganhou importância e se justifica atualmente - e que não se baseiam na melhora ou piora de desfechos. Há motivação ética baseada na autonomia do paciente; argumenta-se, também, que a DC poderia reduzir a variabilidade da prática médica em função apenas do julgamento médico; e, por fim, a DC pode ser vista como essencial para a prática da medicina baseada em evidências, uma vez que esta prática tem em seus fundamentos a consideração de valores e preferências dos pacientes. 
Para médicos de família e comunidade, a DC é uma importante ferramenta de trabalho. Desse modo, conhecer o que motivou seu emprego e ter clareza sobre suas potencialidades e limitações é essencial para seu uso adequado.

\section{Contribuição dos autores}

Concepção e/ou delineamento do estudo: GG, FC.

Aquisição, análise ou interpretação dos dados: GG.

Redação preliminar: GG.

Revisão crítica da versão preliminar: GG, FC

Todos os autores aprovaram a versão final e concordaram com prestar contas sobre todos os aspectos do trabalho.

\section{Conflito de interesses}

Todos os colaboradores negam conflitos de interesses.

\section{Agradecimentos}

Agradeço ao amigo Alexandre Calandrini - com quem passei incontáveis horas discutindo medicina de família e comunidade - pelas contribuições diretas e indiretas para a elaboração deste artigo.

\section{REFERÊNCIAS}

1. Charles C, Gafni A, Whelan T. Shared decision-making in the medical encounter: what does it mean? (or it takes at least two to tango). Soc Sci Med. 1997 Mar;44(5):681-92. DOI: https://doi.org/10.1016/S0277-9536(96)00221-3

2. Makoul G, Clayman ML. An integrative model of shared decision making in medical encounters. Patient Educ Couns. 2006 Mar;60(3):30112. DOI: https://doi.org/10.1016/j.pec.2005.06.010

3. Moumjid N, Gafni A, Brémond A, Carrère MO. Shared decision making in the medical encounter: Are we all talking about the same thing? Med Decis Mak. 2007 Set;27(5):539-46. DOI: https://doi.org/10.1177/0272989X07306779

4. Cribb A, Entwistle VA. Shared decision making: trade-offs between narrower and broader conceptions. Heal Expect. 2011;14(2):210-9. DOI: https://doi.org/10.1111/j.1369-7625.2011.00694.x

5. Beauchamp TL. Methods and principles in biomedical ethics. J Med Ethics. 2003 Set;29(5):269-74. DOI: https://doi.org/10.1136/ jme.29.5.269

6. Conselho Federal de Medicina (CFM). Código de Ética Médica. Resolução CFM n².217, de 27 de setembro de 2018, modificada pelas Resoluções CFM n².222/2018 e 2.226/2019. Brasília (DF): CFM; 2019.

7. Wennberg JE, Barnes BA, Zubkoff M. Professional uncertainty and the problem of supplier-induced demand. Soc Sci Med. 1982;16(7):81124. DOI: https://doi.org/10.1016/0277-9536(82)90234-9

8. Kasper JF, Mulley Junior AG, Wennberg JE. Developing shared decision making programs to improve the quality of health care. Qual Rev Bull. 1992 Jun;18(6):183-90. DOI: https://doi.org/10.1016/S0097-5990(16)30531-0

9. McCormack J, Elwyn G. Shared decision is the only outcome that matters when it comes to evaluating evidence-based practice. BMJ Evidence-Based Med. 2018;23(4):137-9. DOI: https://doi.org/10.1136/bmjebm-2018-110922

10. Stewart M, Brown JB, Weston WW, McWhinney IR, McWilliam CL, Freeman TR. Medicina centrada na pessoa, transformando o método clínico. $3^{\text {rd }}$ ed. Porto Alegre: Artmed; 2017.

11. Kurtz S, Silverman J, Benson J, Draper J. Marrying content and process in clinical method teaching. Acad Med. 2003 Ago;78(8):802-9. DOI: https://doi.org/10.1097/00001888-200308000-00011 
12. Härter M, Moumjid N, Cornuz J, Elwyn G, Van Der Weijden T. Shared decision making in 2017: international accomplishments in policy, research and implementation. Z Evid Fortbild Qual Gesundhwes. 2017 Jun;123:1-5. DOI: https://doi.org/10.1016/j.zefq.2017.05.024

13. National Health Service (NHS). Equity and excellence: liberating the NHS. London, UK: NHS - Department of Health - The Stationery Office Limited; 2010.

14. Australian Commission on Safety and Quality in Health Care (ACSQHC). National Safety and Quality Health Service Standards. $2^{\text {nd }}$ ed. Sydney: ACSQHC; 2017.

15. Ministério da Saúde (BR). Secretaria-Executiva. Núcleo Técnico da Política Nacional de Humanização. HumanizaSUS: Política Nacional de Humanização: a humanização como eixo norteador das práticas de atenção e gestão em todas as instâncias do SUS. Brasília (DF): Ministério da Saúde; 2004.

16. Ministério da Saúde (BR). Portaria ํㅡ 2.436, de 21 de setembro de 2017. Aprova a Política Nacional de Atenção Básica, estabelecendo a revisão de diretrizes para a organização da Atenção Básica, no âmbito do Sistema Único de Saúde (SUS). Diário Oficial da União, Brasília (DF), 22 set 2017; Seção 1:68.

17. Scholl I, Van Loon MK, Sepucha K, Elwyn G, Légaré F, Härter M, et al. Measurement of shared decision making - a review of instruments. Z Evid Fortbild Qual Gesundhwes. 2011;105(4):313-24. DOI: https://doi.org/10.1016/j.zefq.2011.04.012

18. Katz SJ, Hawley S. The value of sharing treatment decision making with patients: expecting too much?. JAMA. 2013 Out;310(15):155960. DOI: https://doi.org/10.1001/jama.2013.278944

19. Souza MT, Silva MD, Carvalho R. Revisão integrativa: o que é e como fazer Integrative review. Einstein. 2010 Jan/Mar;8(1):102-8.

20. Sanders ARJ, Van Weeghel I, Vogelaar M, Verheul W, Pieters RHM, Wit NJ, et al. Effects of improved patient participation in primary care on health-related outcomes: a systematic review. Fam Pract. 2013 Ago;30(4):365-78. DOI: https://doi.org/10.1093/fampra/cmt014

21. Johnson RA, Huntley A, Hughes RA, Cramer H, Turner KM, Perkins B, et al. Interventions to support shared decision making for hypertension: a systematic review of controlled studies. Heal Expect. 2018 Set;21(6):1191-207. DOI: https://doi.org/10.1111/hex.12826

22. Cooper LA, Roter DL, Carson KA, Bone LR, Larson SM, Miller ER, et al. A randomized trial to improve patient-centered care and hypertension control in underserved primary care patients. J Gen Intern Med. 2011;26:1297-304. DOI: https://doi.org/10.1007/s11606011-1794-6

23. Tinsel I, Buchholz A, Vach W, Siegel A, DürkT, Buchholz A, et al. Shared decision-making in antihypertensive therapy: a cluster randomised controlled trial. BMC Fam Pract. 2013 Set;14:135. DOI: https://doi.org/10.1186/1471-2296-14-135

24. Krones T, Keller H, Sönnichsen A, Sadowski EM, Baum E, Wegscheider K, et al. Absolute cardiovascular disease risk and shared decision making in primary care: a randomized controlled trial. Ann Fam Med. 2008;6(3):218-27. DOI: https://doi.org/10.1370/afm.854

25. Loh A, Leonhart R, Wills CE, Simon D, Härter M. The impact of patient participation on adherence and clinical outcome in primary care of depression. Patient Educ Couns. 2007 Jan;65(1):69-78. DOI: https://doi.org/10.1016/j.pec.2006.05.007

26. Loh A, Simon D, Wills CE, Kriston L, Niebling W, Härter M. The effects of a shared decision-making intervention in primary care of depression: a cluster-randomized controlled trial. Patient Educ Couns. 2007 Ago;67(3):324-32. DOI: https://doi.org/10.1016/j.pec.2007.03.023

27. Clever SL, Ford DE, Rubenstein LV, Rost KM, Meredith LS, Sherbourne CD, et al. Primary care patients' involvement in decision-making is associated with improvement in depression. Med Care. 2006 Mai;44(5):398-405. DOI: https://doi.org/10.1097/01.mlr.0000208117.15531.da

28. Ling BS, Trauth JM, Fine MJ, Mor MK, Resnick A, Braddock $\mathrm{CH}$, et al. Informed decision-making and colorectal cancer screening: is it occurring in primary care?. Med Care. 2008 Set;46(9):S23-9. DOI: https://doi.org/10.1097/MLR.0b013e31817dc496

29. Parchman ML, Zeber JE, Palmer RF. Participatory decision making, patient activation, medication adherence, and intermediate clinical outcomes in type 2 diabetes: a STARNet study. Ann Fam Med. 2010;8(5):410-7. DOI: https://doi.org/10.1370/afm.1161

30. LeBlanc A, Herrin J, Williams MD, Inselman JW, Branda ME, Shah ND, et al. Shared decision making for antidepressants in primary care a cluster randomized trial. JAMA Intern Med. 2015 Nov;175(11):1761-70. DOI: https://doi.org/10.1001/jamainternmed.2015.5214

31. Wilkes S, Hall N, Crosland A, Murdoch A, Rubin G. Patient experience of infertility management in primary care: an in-depth interview study. Fam Pract. 2009 Ago;26(4):309-16. DOI: https://doi.org/10.1093/fampra/cmp039

32. World Health Organization (WHO). Mental health - Action plan 2013-2020. Genebra: WHO; 2013.

33. Joosten EAG, DeFuentes-Merillas L, De Weert GH, Sensky T, Van Der Staak CPF, De Jong CAJ. Systematic review of the effects of shared decision-making on patient satisfaction, treatment adherence and health status. Psychother Psychosom. 2008;77:219-26. DOI: https://doi.org/10.1159/000126073 
34. Shay LA, Lafata JE. Where is the evidence? A systematic review of shared decision making and patient outcomes. BMC Med Decis Mak. 2015 Out;35(1):114-31. DOI: https://doi.org/10.1177/0272989X14551638

35. Hughes TM, Merath K, Chen Q, Sun S, Palmer E, Idrees JJ, et al. Association of shared decision-making on patient-reported health outcomes and healthcare utilization. Am J Surg. 2018 Jul;216(1):7-12. DOI: https://doi.org/10.1016/j.amjsurg.2018.01.011

36. Abreu MM, Mello JPS, Ribeiro LFF, Mussi LA, Borges MLL, Petroli M, et al. Shared decision making in brazil. Concrete efforts to empowering patient's voice. Z Evid Fortbild Qual Gesundhwes. 2017 Jun;123:21-2. DOI: https://doi.org/10.1016/j.zefq.2017.05.022 